\section{Promising 3D in vitro models for studying tumour heterogeneity and testing novel therapeutic approaches in pancreatic cancer}

\author{
Stefania Forciniti, 1,2 Marta Cavo, \\ Ilaria Dando, ${ }^{2}$ Elisa Dalla Pozza, ${ }^{2}$ \\ Marta Palmieri,2 \\ Loretta L. del Mercato ${ }^{1}$
}

1Institute of Nanotechnology, National Research Council (CNR-NANOTEC), Lecce; ${ }^{2}$ Department of Neurosciences, Biomedicine and Movement Sciences, Biochemistry Section, University of Verona, Italy

\begin{abstract}
In this study we produced $3 \mathrm{D}$ organotypic cultures and spheroids to mimic the complex microenvironment of pancreatic cancer and to test alternative therapeutic strategies.
\end{abstract}

\section{Introduction}

Pancreatic Ductal Adenocarcinoma (PDAC) is a highly lethal disease with an extremely poor diagnosis and prognosis. ${ }^{1}$ Its aggressiveness is driven by an intense fibrotic desmoplastic reaction in which the increasingly collagen I-rich extracellular matrix (ECM) and several cell types, including Cancer Stem Cells (CSCs), cancer-associated fibroblasts (CAFs) and immune cells create a tumour-supportive environment. ${ }^{2}$ Gemcitabine (GEM) is used as the gold standard drug in PDAC treatment. ${ }^{3}$ However, due to its poor efficacy, it remains urgent to identify novel strategies to overcome resistance issues. In this context, the development of in vitro models that recapitulate the in vivo heterogeneity of the PDAC may be more successful in predicting the efficacy of novel anticancer drugs. ${ }^{4}$

\section{Materials and Methods}

We used three-dimensional (3D) pancreatic cancer models, in particular organotypic cultures grown on an extracellular matrix composed of Matrigel or collagen I to test the effect of the new potential therapeutic prodrug 4-(N)-stearoyl-GEM, called C18GEM. We analysed C18GEM cytotoxic activity and cell inhibition mechanisms induced by the drug on Panc1 cells and the derived CSCs. In a different approach, we generated highly stable 3D tumour spheroids using MiaPaCa 2 cell line by comparing different culture methods. We characterized the growth of $\mathrm{MiaPaCa} 2$ spheroids through morphometric analysis (dimension and aspect ratio), live/dead assay and we designed their molecular features through qPCR analysis of proliferation, mesenchymal and cancer stem cellrelated genes.

\section{Results}

We demonstrated that C18GEM is more effective than the standard treatment with GEM on Panc1 cells and even more on CSCs when cultured in both two-dimensional (2D) and 3D conditions (Figure 1), especially on collagen I. Furthermore, C18GEM induced an increase in cell death and stimulated protective autophagy in Panc1 and CSCs cultured on 3D conditions. 5

In addition, we produced MiaPaCa-2 pancreatic tumour spheroids that show a critical volume, can be easily manipulated and maintain a vital activity (Figure 2 ) and gene expression patterns for many days. ${ }^{5}$

\section{Discussion and Conclusions}

The main features of PDAC are a dense desmoplastic/stromal reaction and a great tumour heterogeneity that contribute to the poor outcome and resistance to therapy. In recent years, significant advances allow the application of 3D-platforms suitable for studying the tumour microenvironment by using tumour cells and scaffolds or matrix, and for identifying effective anti-cancer

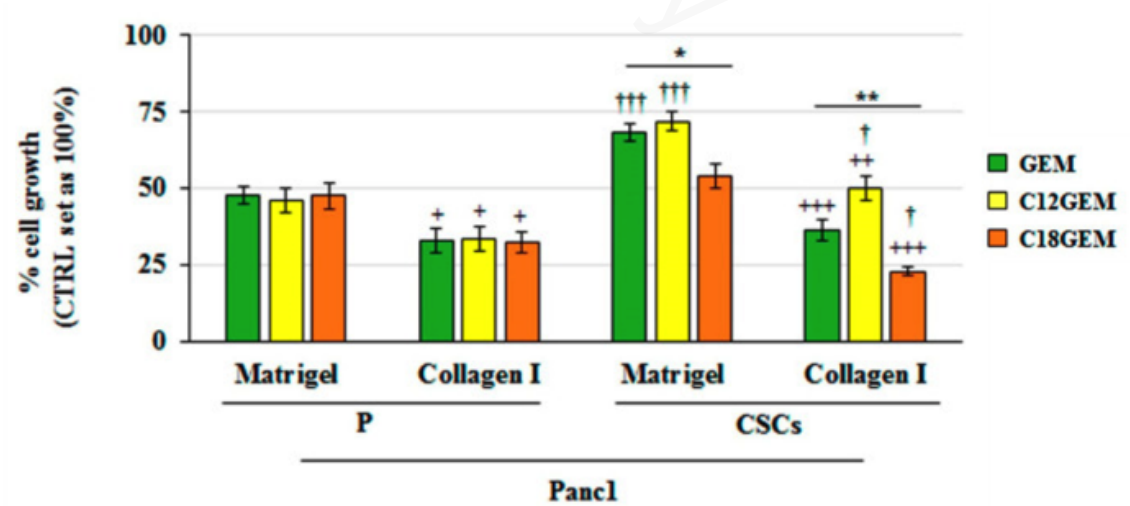

Figure 1. Cell viability analysis of Panc1 cells and CSCs treated with GEM or C12-GEM or C18-GEM for 7 days in Matrigel- and Collagen I-rich extracellular matrix. Values are the means of three independent experiments. Statistical legend: ${ }^{*}$ GEM versus C12GEM or C18GEM and C12GEM versus C18 GEM; + refers to growth on collagen I versus Matrigel for each drug; $\dagger$ CSCs versus parental (P) cells in the two ECMs for each treatment. Adapted from Forciniti et al. ${ }^{5}$
Correspondence: Stefania Forciniti, Institute of Nanotechnology, National Research Council (CNR-NANOTEC), Lecce; Department of Neurosciences, Biomedicine and Movement Sciences, Biochemistry Section, University of Verona, Italy.

E-mail: stefania.forciniti@nanotec.cnr.it

Key words: 3D tumour models; cancer stem cells; tumour microenvironment; pancreatic ductal adenocarcinoma.

Acknowledgments: The authors are grateful to the European Research Council (ERC) under the European Union's Horizon 2020 research and innovation programme (grant agreement No. 759959, INTERCELLMED). This research was partial supported by Joint Projects program 2017 from University of Verona and by the European Research Council (ERC) under the European Union's Horizon 2020 research and innovation programme (grant agreement No 759959, ERC-StG "INTERCELLMED").

Disclosures: The authors declare no conflict of interest.

Conference presentation: This paper was presented at the Third Centro 3R Annual Meeting - L'era delle 3R: modelli in silico, in vitro e in vivo per promuovere la ricerca traslazionale 30 September - 1 October 2021, Evento online organizzato dal Politecnico di Torino.

Accepted for publication: 7 September 2021 .

This work is licensed under a Creative Commons Attribution NonCommercial 4.0 License (CC BY-NC 4.0).

CCopyright: the Author(s), 2021

Licensee PAGEPress, Italy

Biomedical Science and Engineering 2021; 4(s1):169 doi:10.4081/bse.2021.169
Received for publication: 9 July 2021 


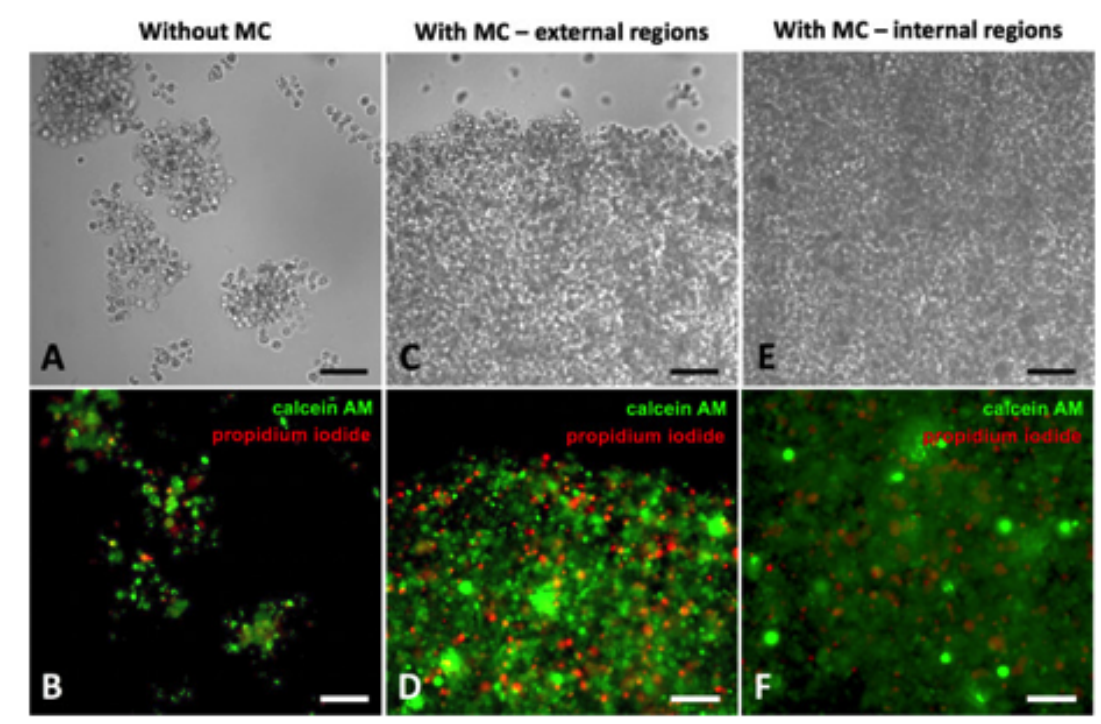

Figure 2. Live/dead assay on spheroids grown with hanging drop. Representative CLSM images of hanging drop-based spheroids without MC (A,B) and with MC (C,D external regions; $\mathrm{E}, \mathrm{F}$ internal regions) stained for live (calcein $\mathrm{AM} ; 3 \mu \mathrm{M}$ solution; in green) and dead (propidium iodide; $10 \mu \mathrm{M}$ solution; in red) cells after 14 days. Bars $=100 \mu \mathrm{m}$. Adapted from Cavo et al. 6

drugs under in vivo-like conditions. However, neither important advancements nor new therapeutic strategies have significantly impacted patient survival and prognosis, highlighting the need to develop in vitro $3 \mathrm{D}$ patient-derived cancer models that allow a personalized drug screening. We propose $3 \mathrm{D}$ pancreatic cancer models, organotypic cultures and spheroids, that may be used as in vitro systems for effective anticancer drug screening. Therefore, the $3 \mathrm{D}$ in vitro systems presented here, can be used as predictive models of patients' response to treatments directing towards the precision medicine in the field of PDAC research.

\section{References}

1. Siegel RL, Miller KD, Jemal A. Cancer statistics, 2020. CA Cancer J Clin 2020;70:7-30.

2. Whatcott CJ, Diep CH, Jiang P, et al. Desmoplasia in Primary Tumors and Metastatic Lesions of Pancreatic Cancer. Clin Cancer Res 2015;21:35618.

3. Lambert A, Schwarz L, Borbath I, et al. An update on treatment options for pancreatic adenocarcinoma. Ther Adv Med Oncol. 2019 Sep 25;11:17588359 19875568.

4. Delle Cave D, Rizzo R, Sainz B Jr, et al. The revolutionary roads to study cellcell interactions in $3 \mathrm{D}$ in vitro pancreatic cancer models. Cancers (Basel) 2021;13:930

5. Forciniti S, Dalla Pozza E, Greco MR, et al. Extracellular matrix composition modulates the responsiveness of differentiated and stem pancreatic cancer cells to lipophilic derivate of gemcitabine. Int J Mol Sci 2020;22:29.

6. Cavo M, Delle Cave D, D'Amone E, et al. A synergic approach to enhance long-term culture and manipulation of MiaPaCa-2 pancreatic cancer spheroids. Sci Rep 2020;10:10192. 